\title{
A novel multiple FISH array for the detection of genetic aberrations in cancer
}

\author{
Ruiyun $\mathrm{Li}^{1}$, Zhenqiu $\mathrm{Liu}^{2}$, Tao $\mathrm{Fan}^{3}$ and Feng Jiang ${ }^{3}$ \\ ${ }^{1}$ Department of Surgery, The University of Maryland School of Medicine, Baltimore, MD, USA; ${ }^{2}$ Department \\ of Biostatistics, The University of Maryland School of Medicine, Baltimore, MD, USA and ${ }^{3}$ Department of \\ Pathology, The University of Maryland School of Medicine, Baltimore, MD, USA
}

\begin{abstract}
Interphase multicolor fluorescence in situ hybridization (IM-FISH) has great promise for improving cancer diagnosis because it can directly visualize multiple changes in chromosomes and gene copy number on a cellto-cell basis. However, no more than four targets can be detected simultaneously by current commercially available IM-FISH protocols, and the DNA probes used are too large to detect the single-gene aberrations that characterize tumorigenesis. As a result, multiple FISH has a low sensitivity in detecting cancer cells. To overcome such limitations, we first developed specific genomic probes for the genes relevant to primary lung cancer. We next designed a multiple FISH array by arranging four different compositions of cocktails of four probes for each gene on a coverslip, which allowed four four-color FISH experiments to be performed in parallel on a single slide. We then tested the multiple FISH array on bronchial brushing samples from lung cancer patients to determine its ability to detect genetic abnormalities. A comparison of the data with the results of cytology and commercial four-color FISH suggested that the multiple FISH array had the highest sensitivity for cancer detection. The technique may thus be a powerful laboratory strategy for cancer prevention and early detection and for improved patient management.
\end{abstract}

Laboratory Investigation (2006) 86, 619-627. doi:10.1038/labinvest.3700408; published online 6 March 2006

Keywords: FISH; DNA probes; diagnosis; in situ hybridization; lung cancer

Carcinogenesis is a heterogeneous disease, in that a single sampled area may not represent the most aggressive portion of a tumor, and rare cellular events may indicate either early pathologic neoplastic lesions or minimal residual disease. ${ }^{1}$ Thus, the diagnostic technologies to analyze individual cells in situ would in theory lead to more accurate diagnosis of the disease. Furthermore, because of the complex and multistep developmental processes of tumorigenesis, diagnostic efforts should have been directed toward developing methods of simultaneously measuring a panel of biomarkers, rather than a single one, to predict a clinically significant cancer phenotype with acceptable accuracy. Therefore, a technique that can be used directly on clinical specimens and can simultaneously measure a panel of biomarkers would represent an advance in diagnosis.

Correspondence: Dr F Jiang, MD, Department of Pathology, The University of Maryland School of Medicine, 10 South Pine Street, MSTF 7th floor, Baltimore, MD 21201-1192, USA.

E-mail: fjiang@som.umaryland.edu

Received 5 January 2006; revised and accepted 1 February 2006; published online 6 March 2006
A number of molecular genetic approaches have been developed to detect cancer cells in various types of specimens. For example, microsatellite, mutation, and methylation analyses have been evaluated extensively in the diagnosis of cancer. ${ }^{2}$ However, these techniques do not address the technical issues specific to the detection of cancer cells in situ and, thus, cannot be used directly on clinical specimens. Therefore, the data obtained from such assays do not represent sample heterogeneity or detect small populations of abnormal cells that may have characteristics, indicating the initiation or progression of cancer. Although microarray analysis of more than 10000 genes simultaneously is promising for the identification of critical genes underlying cancer progression, it is limited in its reproducibility, accuracy, and cost-effectiveness and is labor intensive. In addition, although antigenbased methods, such as immunohistochemistry for identifying proteins, can be performed in situ, they can only detect a single antibody at a time and have excessively low sensitivity. Therefore, these techniques have not been proven to be sufficiently useful in a clinical setting for the early detection of cancer.

More promising is the recently designed interphase multicolor fluorescence in situ hybridization 
(IM-FISH) technique. IM-FISH reveals cell-to-cell heterogeneity, can detect minor subpopulations of genetically distinct cells, allows examination of specimens for direct visualization of multiple subchromosomal or genomic aberrations, and does not require microdissection or DNA and RNA preparation. ${ }^{3}$ Furthermore, compared with other techniques, IM-FISH is more quantitative and requires fewer samples. In addition, unlike metaphase-based multiple FISH, IM-FISH does not require cell culture and chromosomal preparations. However, the current commercially available IM-FISH protocols have some drawbacks. For example, no more than four genomic targets can be detected simultaneously, and DNA probes targeting large genomic fragments are too large to detect the single-gene deletions and amplifications that characterize the early stages of carcinogenesis. Therefore, although the sensitivity and specificity of these IM-FISH tests are superior to those of some conventional diagnostic tools, the false-negative and false-positive rates are still unacceptably high in the detection of genetic abnormalities specific to the phenotype of cancer cells. ${ }^{4}$

Basing our design on array techniques and taking advantage of in situ hybridization and multicolor labeling, we developed a multiple FISH array that cannot only be used directly on clinical specimens but can also simultaneously detect a panel of unique genetic aberrations with multiple specific probes. We first generated gene-specific genomic probes for the most clinicopathological relevant genetic signatures in primary lung cancer and designed a multiple FISH array consisting of the probes. We then tested the array on bronchial brushings from lung cancer patients to detect genetic abnormalities and compared the data with results of cytologic studies and of a commercial four-color FISH set probes. We proved that this multiple FISH array offered the highest sensitivity for cancer detection, despite the heterogeneity of lung tumorigenesis. We concluded that the technique might be a practical, costeffective strategy in the laboratory setting for prevention and early detection of cancer and for improved patient management.

\section{Materials and methods}

\section{Patient and Control Population and Specimen Preparation}

Forty patients with stage I nonsmall lung cancer were recruited in the study. All patients had a lung mass that was operable. The patients underwent bronchoscopy just before surgical excision. Bronchial brushings were taken from the mainstem bronchus on the tumor-bearing side. Normal lymphocytes from six healthy individuals and bronchial washings from 20 individuals with no history of smoking or lung cancer were used as controls. Cell suspensions of bronchial washings were dropped onto the slides and fixed in Carnoy's solution (methanol to acetic acid, 3:1) for $20 \mathrm{~min}$ and then stored in freezer at $-80^{\circ} \mathrm{C}$.

\section{Designing a Cancer-Specific Genomic Probe Panel}

A panel of eight specific genomic probes and their corresponding chromosomal centromeric probes were used to design the multiple FISH array (Table 1). The genes were selected on the basis of our previously published primary lung cancer-specific genomic signatures, which were detected by a microarray-based comparative genomic hybridization analysis. ${ }^{5,6}$ To develop the unique genomic

Table 1 Panel of eight probes and their corresponding chromosomal centromeric probes arrayed on the in situ DNA chip

\begin{tabular}{|c|c|c|c|}
\hline Square (element) & Probe name and chromosomal location & DNA sequence type & Fluorochrome \\
\hline A & $\begin{array}{l}\text { CEP1, chromosome } 1 \text { centromere } \\
\text { ENO1, 1p36.23 } \\
\text { CEP9, chromosome } 9 \text { centromere } \\
\text { p16, 9p21.3 }\end{array}$ & $\begin{array}{l}\text { Alpha satellite DNA } \\
\text { BAC DNA } \\
\text { Alpha satellite DNA } \\
\text { LD-DOP-PCR DNA }\end{array}$ & $\begin{array}{l}\text { Texas red (Qbiogene) } \\
\text { Alexa Fluor } 555 \text { (yellow) } \\
\text { Aqua (Vysis) } \\
\text { Alexa Fluor } 514 \text { (green) }\end{array}$ \\
\hline $\mathrm{B}$ & $\begin{array}{l}\text { CEP3, chromosome } 3 \text { centromere } \\
\text { GC20, 3p22.1 } \\
\text { CEP11, chromosome } 11 \text { centromere } \\
\text { FGF4, 11q13.3 }\end{array}$ & $\begin{array}{l}\text { Alpha satellite DNA } \\
\text { LD-DOP-PCR DNA } \\
\text { Alpha satellite DNA } \\
\text { LD-DOP-PCR DNA }\end{array}$ & $\begin{array}{l}\text { Texas red (Qbiogene) } \\
\text { Alexa Fluor } 555 \text { (yellow) } \\
\text { Aqua (Vysis) } \\
\text { Alexa Fluor } 514 \text { (green) }\end{array}$ \\
\hline C & $\begin{array}{l}\text { CEP8, chromosome } 8 \text { centromere } \\
Y W H A Z, 8 \mathrm{q} 23.1 \\
C E P 10 \text {, chromosome } 10 \text { centromere } \\
S P-A, 10 \mathrm{q} 22.3\end{array}$ & $\begin{array}{l}\text { Alpha satellite DNA } \\
\text { BAC DNA } \\
\text { Alpha satellite DNA } \\
\text { BAC DNA }\end{array}$ & $\begin{array}{l}\text { Texas red (Qbiogene) } \\
\text { Alexa Fluor } 555 \text { (yellow) } \\
\text { Aqua (Vysis) } \\
\text { Alexa Fluor } 514 \text { (green) }\end{array}$ \\
\hline $\mathrm{D}$ & $\begin{array}{l}\text { CEP7, chromosome } 7 \text { centromere } \\
\text { COL1A2, 7q21.3 } \\
\text { CEP15, chromosome } 15 \text { centromere } \\
\text { ANXA2, } 15 q 22.2\end{array}$ & $\begin{array}{l}\text { Alpha satellite DNA } \\
\text { BAC DNA } \\
\text { Alpha satellite DNA } \\
\text { BAC DNA }\end{array}$ & $\begin{array}{l}\text { Texas red (Qbiogene) } \\
\text { Alexa Fluor } 555 \text { (yellow) } \\
\text { Aqua (Vysis) } \\
\text { Alexa Fluor } 514 \text { (green) }\end{array}$ \\
\hline
\end{tabular}


probes that would specifically cover the genomic sequences of the selected genes, we used two strategies. First, for the genes with a large genomic length that potentially showed increased copy number changes in lung cancer cells, we searched several gene databases, including the Celera database (http://www.celera.com) and the Ensembl human genome database (http://www.ensembl.org) to identify BAC or PAC clones containing only the genomic sequences of the genes of interest. After obtaining the relevant clones (Invitrogen Corporation, Carlsbad, CA, USA), we prepared inset DNA to use as a probe, as described previously. ${ }^{7}$

Second, to develop a specific genomic probe that exactly covered the full length of the genome for the genes with a small genomic size, especially those potentially deleted in cancer cells, we first identified clones that might contain genomic sequences of several genes, including the specific gene of interest. We then amplified considerably larger (1-10 kb) amplimers by long-distance PCR (LD-PCR) with specific primers for the target gene from the clone DNA. With this approach, several overlapping bidirectional PCRs with structurally complex genomic sequences were produced to span the whole genomic region of the target gene. The DNA of the LD-PCR amplicons was then amplified by degenerate oligonucleotide-primed PCR (DOP-PCR) (DOP PCR Master; Roche Diagnostics Corporation, Indianapolis, IN, USA), according to the manufacturer's instruction. ${ }^{8}$ Finally, the DOP-PCR-amplified DNA was labeled by a nick translation reaction with fluorochrome-dUTP (Alexa Fluor; Molecular Probes, Eugene, OR, USA) (Table 1), as described previously. $^{{ }^{7}}$ The hybridization efficiency of each probe was tested and validated on the chromosomal spreads of normal lymphocytes and together with their corresponding chromosomal centromeric probes (Vysis Inc., and Qbiogene, Irvine, CA, USA), which were used as controls. To further validate the size and the mapping region of the gene-specific genomic probes developed by the LD-PCR and DOPPCR protocols, segments of the DNA products and final probe were analyzed on normal DNA fiber preparations, as described previously. ${ }^{9}$

\section{Designing and Manufacturing the Multiple FISH Array}

The multiple FISH array consisted of 16 probes, including the gene-specific probes and their corresponding chromosomal centromeric probes. Four different compositions of cocktails of four probes each were arrayed (Figure 1 and Table 1). These four probes were labeled in red, green, aqua, and yellow (Table 1). All the chromosomal centromeric probes were commercially available (Vysis Inc., and Qbiogene).

One side of a conventional $36 \times 36-\mathrm{mm}$ glass coverslip was divided into four separate squares of equal size by mounting on a high-melt agarose gel (Figure 1); each square was $8 \times 8 \mathrm{~mm}$ and marked as square A, B, C, or D on the backside of the coverslip to indicate its location. The distance between each square was $6 \mathrm{~mm}$. Four different cocktails of probes were precipitated with ethanol. Each cocktail contained $100 \mathrm{ng}$ of each probe mixed with a 30 -fold excess of human Cot-1 DNA (Life Technologies, Rockville, MD, USA) and was resuspended after precipitation in $6 \mu$ l of a matrix of glass-immobilized gel elements by polymerization of a $20-\mathrm{mm}$-thin $8 \%$ polyacrylamide gel (Sigma-Aldrich, Saint Louis, MO, USA); this polymerization reversibly bound each square on the glass coverslip (Figure 1). After $10 \mathrm{~min}$, the matrix of glass-immobilized gel elements containing the probes became solid at room temperature, whereas the gel remained soluble and allowed the probes to hybridize on the target cells at a temperature of $37^{\circ} \mathrm{C}$ or above. However, the separate high-melt agarose gel did not melt at $100^{\circ} \mathrm{C}$, which prevented the mixing of probes from different squares during denaturation and hybridization.

Slides with mononuclear cells were mounted in a 20- $\mu$ l hybridization buffer (Vysis) and then were covered by inverting over the multiple FISH array, which was positioned upside down (Figure 1). After the edge of the coverslip was sealed with rubber cement (Sigma-Aldrich), the location of each square was also marked on the backside of the slide with a diamond pen, corresponding to the previously marked location on the backside of the coverslip. The hybridization was performed on the HYBriteTM Denaturation/Hybridization unit (Vysis) according to the manufacturer's instructions with the following program: denaturing at $74^{\circ} \mathrm{C}$ for $4.5 \mathrm{~min}$ and hybridization at $37^{\circ} \mathrm{C}$ overnight. Postwashing was performed under the conventional FISH protocol described in our previously published study. ${ }^{7,9}$ Finally, the cells were counterstained with $0.2 \mu \mathrm{M}$ of $4^{\prime}, 6$-diamidino-2-phenylindole (DAPI).

In addition, to test whether the separation methodologies used and the space between each element of the array design would prevent the mixing of probes from different elements during hybridization, the centromeric probes for chromosomes 1 (in red; Qbiogene), 2 (in green; Qbiogene), 3 (in aqua; Vysis), and 4 (in orange; Vysis) were added to each square of the coverslip. These probes were then hybridized on five normal control and five cancer specimens. To evaluate the performance of the array, we also tested a commercial LAVysion four-color probe set (Vysis) that targeted centromere 6, 5p15.2, 7p12 (EGFR), and 8q24 (MYC) on the same specimens in parallel using the same protocol described in our previous study. ${ }^{4}$ For cytologic diagnosis, Papanicolaou-stained preparations were also made from each bronchial washing specimen in parallel with the slides fixed in Carnoy's solution. 

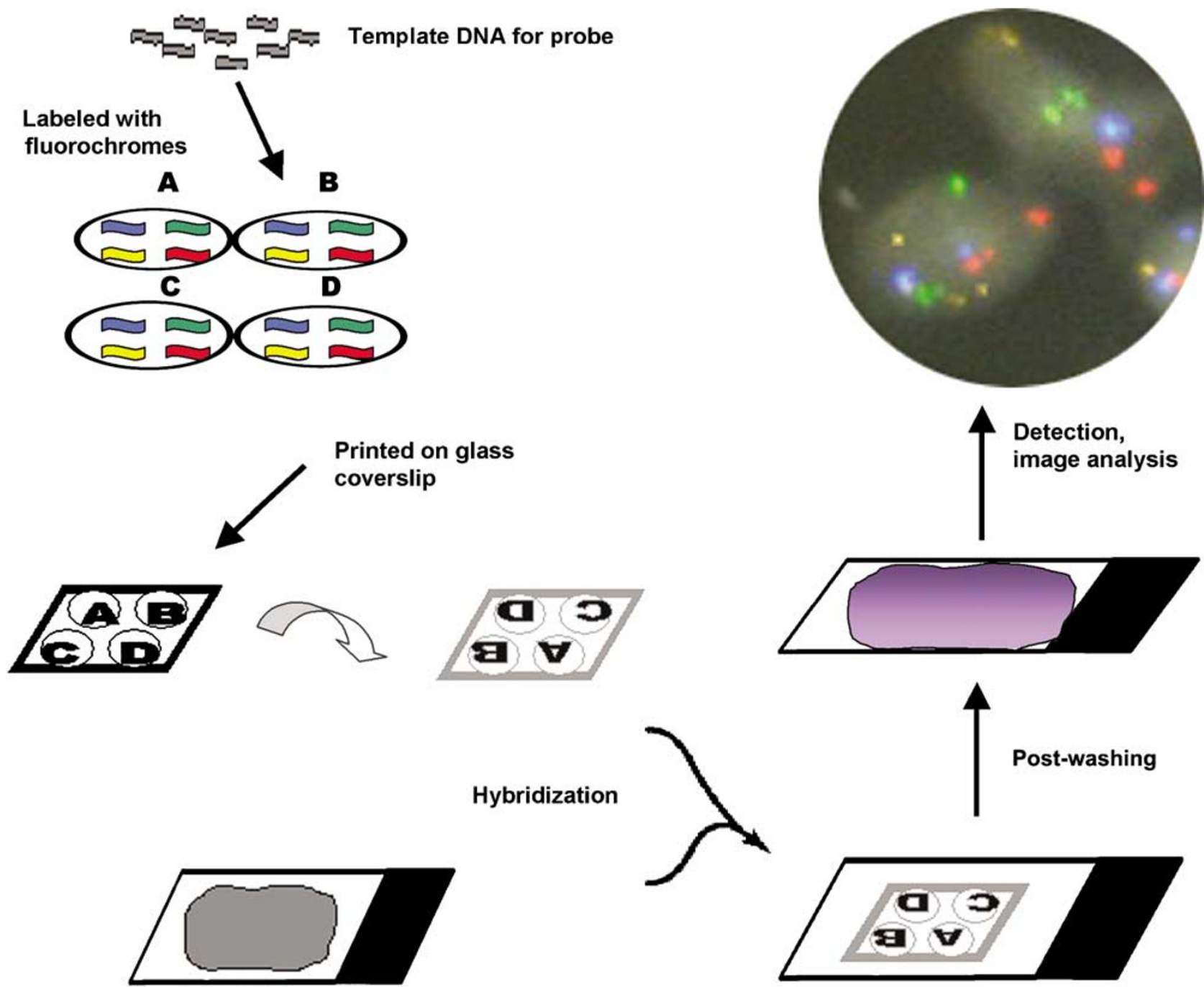

Hybridization
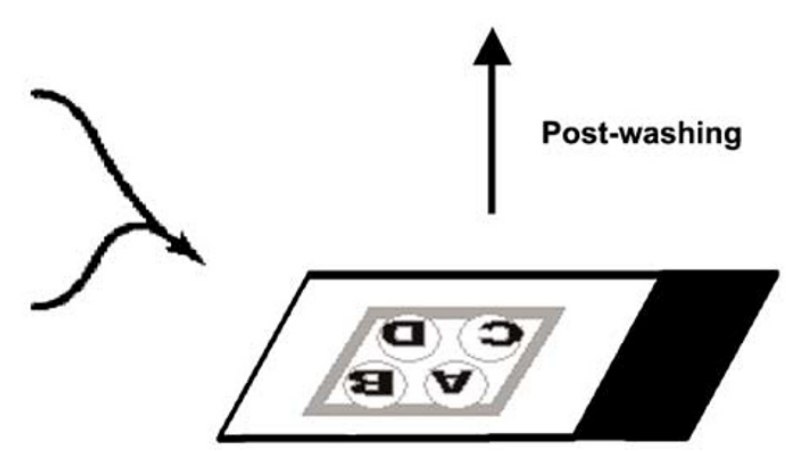

Figure 1 Multiple FISH array schema. The template DNA of the genes of interest was labeled with four fluorochromes, and the probes were arranged in four elements. Each element contained a cocktail of four probes and then printed on a coated glass coverslip. After the hybridization, solution was added to the slide, and the coverslip was inverted over the slide. Four corresponding squares were marked on the back of the slide, according to the location of the four elements on the coverslip. After hybridization and postwashing, signals were counted and images captured, based on the marked line on the backside of the slide.

\section{Epifluorescence Microscopy}

Images from the multiple FISH array and LAVysion probe set assays were obtained with epifluorescence microscopy (Leica Microsystems Inc., Bannockburn, IL, USA). The microscope was equipped with a filter set (Chroma Technology Corp., Brattleboro, VT, USA) that included DAPI, aqua, yellow, red, and green single band passes, which were specific for the four spectrally distinct fluorochromes used for the probes in the multiple FISH array and LAVysion probe set assay. To count the signals from the LAVysion probe set, at least 100 cells were analyzed, as described previously. ${ }^{4}$ The signals from the multiple FISH array were counted in a similar manner, and the number of aberrations per 100 interphases per square was recorded from square A through square D serially, according to the marked line on the backside of the slides.

We used two criteria for considering a cell abnormal: (1) the cell has three or more copies or exhibits hemizygous or homozygous loss of a specific gene probe signal; and (2) although having no loss of any specific gene probe, as described in the first criterion, the cell has an aneusomy (such as monosomy, trisomy, or tertrasomy) of any centromeric probe.

\section{Statistical Analysis}

The sensitivity and specificity of all assays were calculated by using receiver operating characteristic (ROC) analysis. To be conservative in estimating the percentage of abnormal specimens, we chose as the 
optimal cutoff the one with a $0 \%$ false-positive rate or, equivalently, $100 \%$ specificity. $\chi^{2}$ Tests were used to compare the results of the multiple FISH array with the data generated by the LAVysion multicolor probe set and cytology.

\section{Results}

\section{Generation and Validation of the Hybridization} Efficiency of the Gene-Specific Genomic Probes

The specific signals of all the counted metaphases of each probe were labeled correctly, and the probes were hybridized in the correct chromosomal location. This was assessed by viewing the individual fluorochrome channels in a karyotype format, which indicated the absence of chimerical signals and the extreme brightness of the signals (Figure 2). The hybridization efficiency (defined as the percentage of cells with fluorescent signals on both sister chromatids of both chromosomal homologs) of the probes on the normal metaphase spreads was $100 \%$, as was the hybridization efficiency of the commercial centromeric probes. There was no overlap of wavelengths among the four spectrally distinct fluorochromes: the signals were clearly distinguishable from each other under the appropriate filters. Furthermore, the exact size and mapping region of each gene-specific probe developed by the LD-DOPPCR protocol was confirmed by FISH on normal DNA fiber, which showed a single linear DNA molecule with juxtaposed color barcode signals (Figure 3).

\section{Performance of the Multiple FISH Array and Definition of its Cutoff Value}

Normal lymphocytes from six healthy individuals displayed two signals (disomy) for each probe, as shown by the normal diploid population that comprised two aqua, two green, two red, and two yellow signals in each element detected. The multiple FISH arrays containing only a one-color fluorescence-labeled probe in each element were also tested in 10 specimens. In all cases, square A showed only a red signal, square B a green signal, and so on, indicating that no mixture of the probes occurred between different squares during hybridization.

To establish the cutoff value that determined whether a case was positive or negative for cancer, we calculated the number of abnormal cells in each specimen. An ROC curve was plotted for sensitivity as a function of $100 \%$ specificity, based on the percentage of abnormal cells. The optimal point in the assay corresponded to a cutoff value of six abnormal cells per specimen. Consequently, a specimen was classified as positive for cancer if it contained six or more abnormal cells; otherwise, the sample was considered negative for cancer.

\section{Detection of Cancer Cells by the Multiple FISH Array and Comparison with Results of Conventional Cytologic and an IM-FISH Tests}

Of the 40 bronchial washing specimens from patients with a histologically confirmed diagnosis
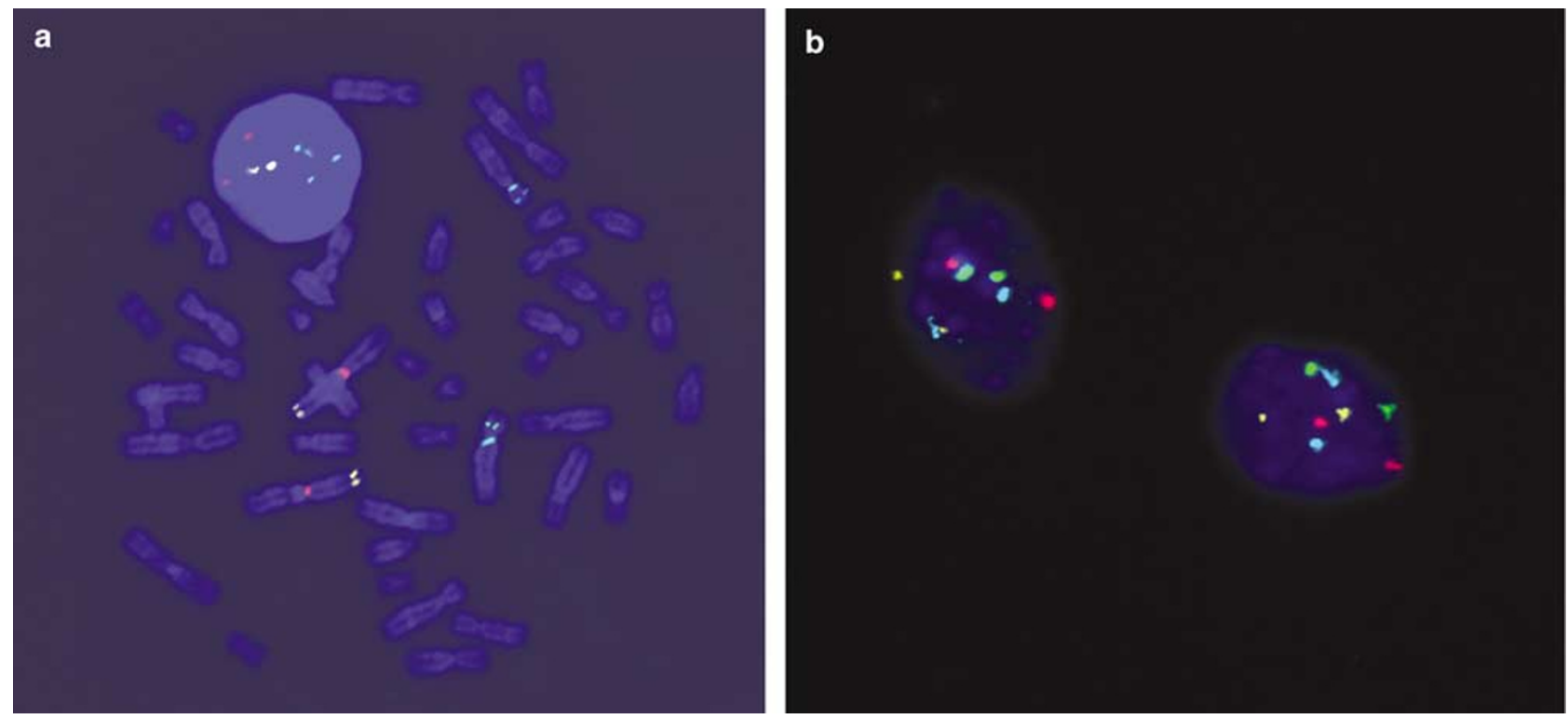

Figure 2 Validation of the gene-specific genomic probes on the chromosomal spreads of normal lymphocytes and normal bronchial epithelial cells and of their corresponding chromosomal centromeric probes used as controls. (a) Chromosomal spread of normal metaphases shows the ENO1 probe on 1p36.23 (yellow), the CEP1 probe on the centromere of chromosome 1 (red), the p16 probe on 9p21.3 (green), and the CEP9 probe (aqua). (b) Normal interphases show two yellow signals of the ENO1 probe, two red signals of CEP1 probe, two green signals of the CEP10, and two aqua signals of the CEP9 probe. 

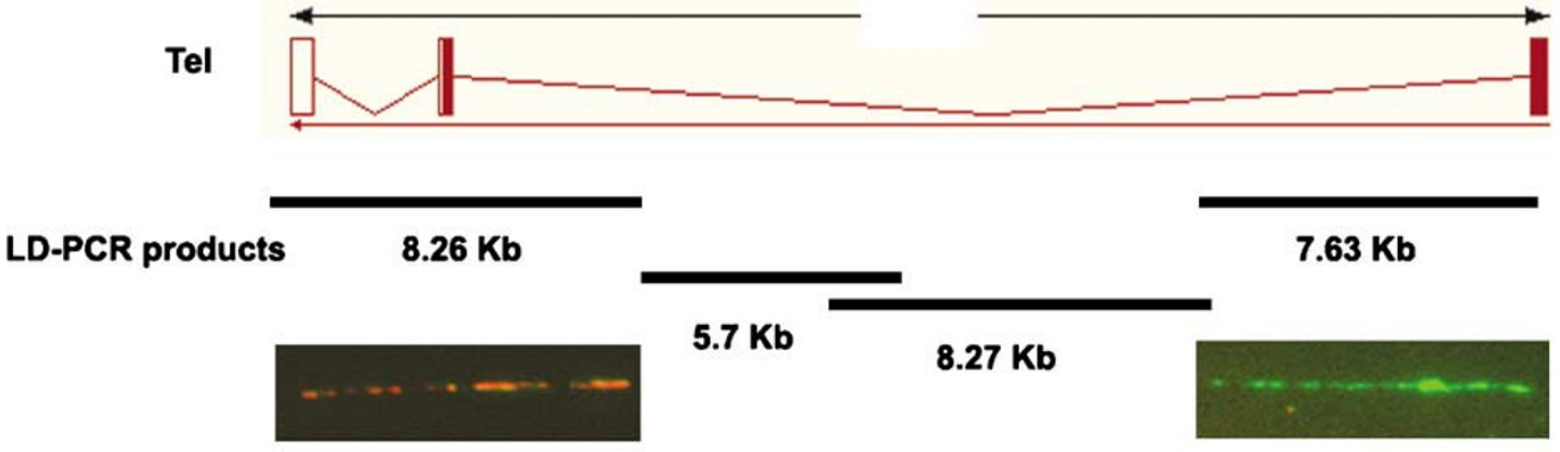

Fiber FISH of LD-PCR products

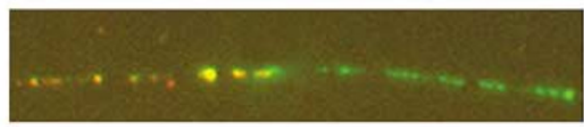

DOP-PCR product (FISH probe)

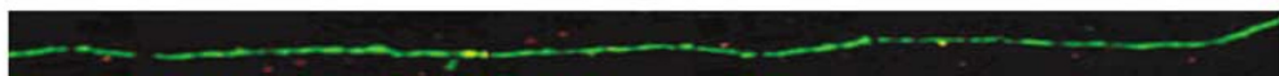

Figure 3 Schematic $p 16$ gene map of normal DNA fiber detected by a gene-specific probe developed with the LD-DOP-PCR strategy. (Top) Genomic structure of $p 16$. The four black bars denote the size and location of LD-PCR products, which were confirmed by the genomic fiber detected by FISH. (Bottom) The green fiber shows the product of DOP-PCR, which is a 27-kb gene FISH probe spanning the entire length of $p 16$.

of lung cancer, 36 were classified as abnormal by the multiple FISH array, indicating that this assay had a $90 \%$ sensitivity in detecting cancer cells. In the cancer-positive samples, the percentage of abnormal cells was variable, ranging from 11 to $78 \%$ (mean, $44.5 \%$ ), which was statistically significantly higher than the $6 \%$ cutoff value $(P=0.008)$. Using the same bronchial washing specimens, cytologic analysis suggested the presence of cancer in $21(52.5 \%)$ cases and IM-FISH (LAVysion) in 26 (67.5\%). Of the 19 cases of cancer not detected by cytologic analysis, the LAVysion kit and our multiple FISH array detected six and 15 cases, respectively, suggesting that the use of molecular genetic diagnostic methods improved the accuracy of the diagnosis of cancer. Furthermore, in the 14 cases of LAVysion-negative specimens, 10 cases were positive according to the multiple FISH array (Figure 4), showing that the sensitivity of the array for detecting cancer cells in bronchial washing specimens was statistically higher than that of either cytology or LAVysion $(P=0.006$ and 0.003 , respectively).

None of the bronchial brushing specimens from the 20 individuals with no history of smoking or lung tumors were shown to have abnormal cells by cytology analysis, the LAVysion kit, or the multiple FISH array, suggesting that the specificity of the three tests was same ( $100 \%$ by design).

\section{Discussion}

We showed that the sensitivity of the multiple FISH array for simultaneously measuring a panel of biomarkers was markedly higher than the sensitivities of cytologic analysis and LAVysion IM-FISH, indicating that our array represents a substantial improvement in the detection of lung carcinoma in bronchial washing specimens. Because the multiple FISH array also had the same specificity as did cytology and LAVysion IM-FISH, it might be a powerful diagnostic test and a useful adjunct to cytologic analysis in the examination of bronchial secretions for the evidence of cancer.

The sensitivity of a genetic assay is directly proportional to the number of probes used simultaneously. As a result, IM-FISH has great promise for increasing the rate of cancer diagnosis at the singlecell level because it allows visualization of multiple probes simultaneously and, thus, streamlines the screening of specimens for chromosomal aneuploidies and/or copy number changes of specific cancerassociated genes. This is particularly important given the completion of the human genome project and more tumor-specific genes being identified. There are currently two approaches for increasing the number of fluorophore-labeled probes used in IM-FISH. First, different fluorescence dyes can be 

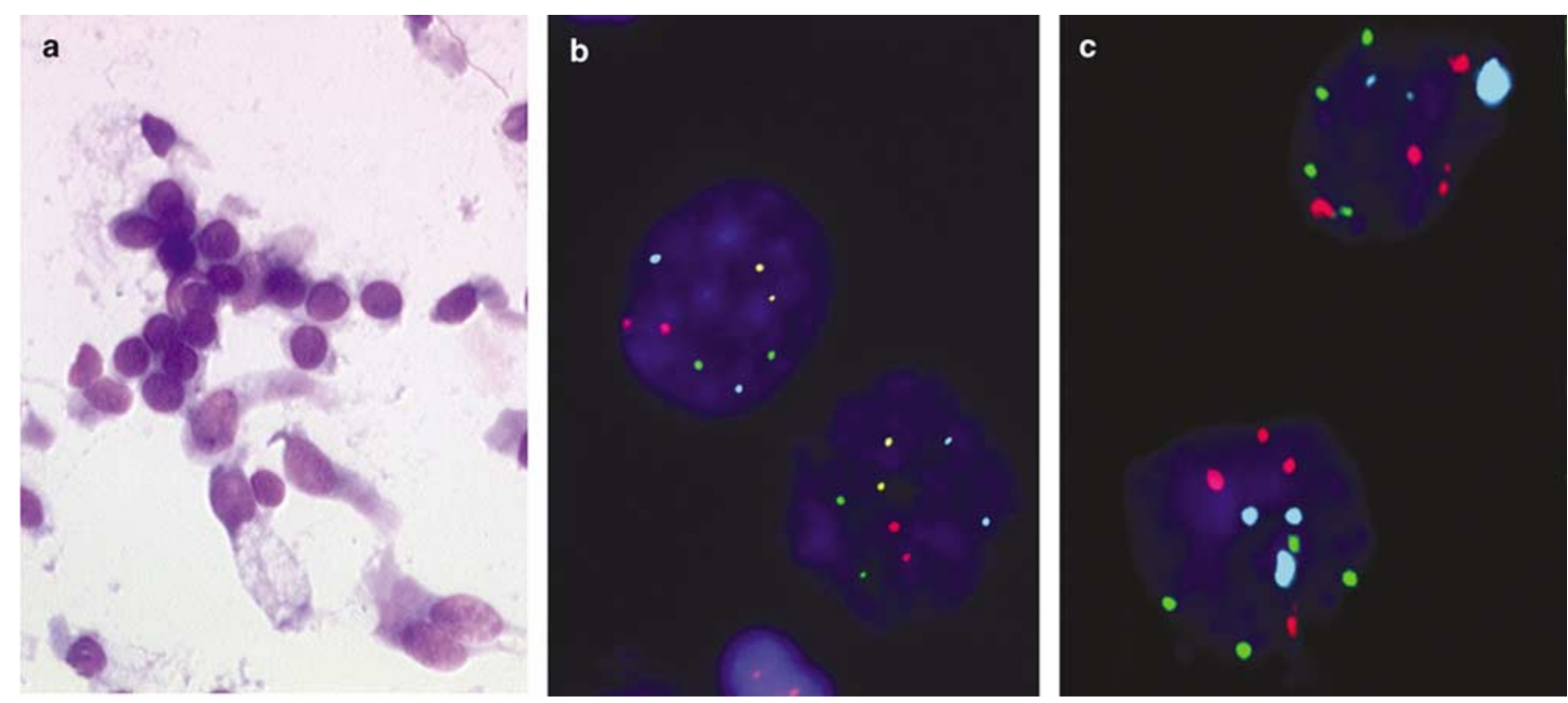

Figure 4 Representative hybridization images of LAVysion-negative and multiple FISH array-positive cancer cells. (a) Bronchial washing sample showing an unremarkable bronchial epithelium in a cytospin preparation (original magnification $\times 60$ ). (b) Results of a LAVysion assay of the bronchial brushing in (a) showing a normal diploid population (original magnification $\times 600$ ). (c) Multiple FISH array results showing genetic aberrations in the cells from the sample in (a) (original magnification $\times 600$ ).

used for either combinatorial labeling or ratio labeling. Although this approach can theoretically distinguish a greater number of targets for a given number of fluorescent labels, it is more complicated in terms of probe labeling, so more accurate fluorescence measurements are needed. For example, if hybridization intensities were poor or background and autofluorescence were high, especially in nuclei, a pair of ratios could be so close that the color difference between the targets would be too small to clearly distinguish between the targets, thus resulting in very high hybridization variability artifacts. The second approach is the least ambiguous and simplest method of multitarget analysis. In this technique, a different, spectrally distinct fluorescent label stains each probe in a single assay. Current epifluorescence microscopy consisting of as many as eight different color channels or filters can be used for the simultaneous analysis of up to eight probes; however, not all eight targets could be visually distinguished with certainty based solely on their color when viewed directly through the microscope using any single bandpass filter sets. For example, probe stains range from green to orange, with the intermediate gradations of yellow and orange difficult to distinguish from one another. Furthermore, even without these concerns, the size of the interphase also does not allow many probes to be hybridized simultaneously. We have found that using a panel of six probes in interphases will result in very labor-intensive screening and great interpretative challenges when signals are counted in highly aneuploid samples; ${ }^{4}$ Furthermore, focal plane distortions can occur because of either overlapping signals or the blending of fluorophores when the different signals were close to one another.
Therefore, it is not worthwhile to simply increase the number of labeling probes arrayed in an interphase molecular cytogenetic diagnostic test.

In this study, rather than merely increasing the number of fluorochromes to achieve a reduction in probe complexity, we proposed instead a conceptual change by which a coverslip was divided in four small subregions, and four-color FISH experiments were performed in parallel. We clearly demonstrated that the unique separation methodologies used and the wide space left between each element of the array design prevented mixing of the probes from different elements during hybridization. Furthermore, to avoid any overlapping of adjacent fluorochromes owing to significant overlap in the excitation and emission spectra, we chose green, red, aqua, and yellow fluorochromes in our array design. These four distinct dyes have the most discriminative wavelengths and have been shown to be the most stable and brightest fluorochromes; this prevented the possibility of overlapping, bleaching, or weakness of the hybridization signals. As expected, in this study all genetic and chromosomal targets were classified correctly in metaphases, interphases, and DNA fiber.

Because the multiple FISH array consists of fewer fluorochromes and requires less labeling, fewer filter sets, and fewer images to be recorded, both the image acquisition and image-processing times are reduced. Furthermore, counting the signals of the multiple FISH array requires only a conventional fluorescent microscope equipped with common fluorescent filters and a digital camera rather than more sophisticated imaging systems and software for performing analyses. This will greatly benefit clinical and research laboratories that lack access to 
the complicated and expensive equipment generally needed to perform molecular genetic studies. In particular, the strategy may satisfy the requirements of speed, simplicity, and reliability demanded of routine diagnostic tools in the clinical setting.

Most current DNA probes are made from large genomic fragments cloned from a variety of vectors, including PAC (80-100 kb), BAC (100-200 kb), and YAC (1-2 mb), and only target subchromosomal and band-specific genomic regions. ${ }^{3}$ Therefore, these probes cannot detect cancer-specific genetic changes, especially single-gene deletions, which are, in most cases, specific genetic events that occur in preneoplastic cells or in the early stages of carcinogenesis. In contrast, we successfully generated specific genomic probes for the cancer-related genomic signatures. Furthermore, given that the size of some specific probes that have been developed are comparatively small, which can lead to falsenegative results in the detection gene deletions because the signals of the genes may be artificially weak, we used Alexa fluorochromes to label the probes. These fluorochromes have been shown to possess superior brightness and photostability compared with conventional fluorochromes and have been successfully used to label cDNA probes. ${ }^{10,11} \mathrm{In}$ fact, we showed that even the smallest genomic probes (eg, $2.3 \mathrm{~kb}$ for the FGF4 probe) labeled with Alexa fluorochromes exhibit very bright signals with high hybridization sensitivity and efficiency compared with commercial centromeric probes in the detection of target genes. These results were also confirmed by the DNA fiber spreads, implying that the generated specific gene probes can overcome the low detection efficiency of conventional large-fragment DNA probes. Therefore, the genomic changes detected by our probes might better reflect the real genotypes of cancer cells. For interphase molecular genetic diagnosis, careful selection of the probe type is another key to provide accurate diagnostic information. The LAVysion kit was designed to identify cells with changes of three selected chromosomal loci $(5 p 15,8 q 24,7 p 12)$ and of the centromeric region of chromosome 1 , which is used as an indicator of the ploidy status of the tested cell; however, no corresponding centromeric probe for chromosomes 5, 7, and 8 are included in the assay kit as controls for these specific chromosomal loci probes. Furthermore, CEP1 is obviously unreliable to verify the copy number of the tested genes located on other chromosomes because it only represents chromosome 1. In contrast, here we designed the probe panel of the multiple FISH array solely on the pattern of genomic signatures in primary lung cancer, as defined by our previous comprehensive microarray-based comparative genomic hybridizations. ${ }^{5,6}$ Our multiple FISH array allows a panel of 16 probes, including eight centromeric probes for enumerating chromosomes, which can more precisely examine the ploidy status (ie, chromosomal aneuploidy) of the cell than can the LAVysion kit, which only detects one chromosome. More importantly, the multiple FISH array can detect eight different cancer-specific genetic changes rather than only the three chromosomal loci and has internal control probes, which further permit specific assessment of the cancer-specific genomic aberrations. Therefore, it not surprising that the sensitivity of the multiple FISH array is not only higher than that of cytologic analysis but also markedly higher than that of four-color LAVysion IM-FISH in the detection of cancer cells.

Our technique differs from an approach that divides a single slide into 24 subregions and involves 24 single- or dual-color FISH experiments performed in parallel. ${ }^{12}$ First of all, the latter procedure requires a sufficient supply of specimen material and so is particularly difficult when clinical samples are limited in number. Furthermore, the technique requires sophisticated imagecapture analysis to ensure that the correct area from the 24 subregions is selected for counting the correct probes on one slide. Our technique also differs from a comparative genomic hybridization-based BAC/PAC microarray in which the hybridization is performed on large DNA fragments immobilized on a matrix. ${ }^{13}$ The microarray consisting of welldefined genomic clones such as BAC or PAC provides complete coverage of some chromosomal arms, which are frequently altered in a variety of cancers. However, like other throughput array techniques, such a microarray cannot test samples in situ. In addition, this microarray provides very low resolution for detecting genomic changes of targets, given that BAC or PAC DNA fragments are approximately $100-200 \mathrm{~kb}$ in length.

The multiple FISH array is most likely to succeed in clinical specimens that can yield single dispersed cells like bronchial washes or other cytology material, however, may not be suitable for small biopsy specimens or in fixed tissues where there is a limited region of neoplasia. This is because the set of 16 independent probes are divided into four discrete regions (squares) of the coverslip, and unless one gets a fortunate 'hit' in one of the quadrants, this platform would result in a false negative. Further modifying the technique including reducing size of each square and distance between each square might solve the problem.

Although the current study demonstrated that the multiple FISH array strategy might have potential as a rapid and reliable screening method for detecting tumor-specific genetic changes in biologic fluid specimens from lung cancer patients, longitudinal validation of the strategy in nest case-control study are needed to establish diagnostic criteria for the detection of cancer cells in easily accessible tissues. In an ongoing study, we are testing the multiple FISH array using sputum and/or bronchial brushing samples from lung cancer patients and cancer-free heavy smokers and nonsmokers to determine the true sensitivity and specificity of the assay. We will 
also follow-up the smokers to identify the genetic alterations that might constitute markers for a risk of developing lung cancer. Furthermore, although we only developed a lung cancer-specific multiple FISH array in this study, our strategy may also have broad and flexible applicability in the risk assessment, early diagnosis, and staging of other types of cancer, especially when combined with other appropriate chromosomal probes or tumor-specific genes. With this in mind, we are developing an automated counting system for signals of the multiple FISH array. Once this counting protocol is established, we expect the efficiency of the assay to be greatly enhanced with high-throughput automation.

\section{Acknowledgements}

We thank Ellen McDonald of the Department of Scientific Publications for editorial review of this manuscript and Dr Zeng Wang of Department of Cardiothoracic Surgery, Central Hospital, Huangyang in China for providing parts of specimens tested in the study. This work was funded by National Cancer Institute Grant CA-113707 (to Feng Jiang).

\section{References}

1 Vogelstein B, Kinzler KW. The multistep nature of cancer. Trends Genet 1993;9:138-141.

2 Dolken G. Detection of minimal residual disease. Adv Cancer Res 2001;82:133-185.

3 Jiang F, Katz RL. Use of FISH as a powerful diagnostic tool in cytology. Diagn Mol Pathol 2002;11:47-57.
4 Barkan GA, Caraway NP, Jiang F, et al. The potential use of FISH to identify predictive markers in earlystage lung carcinomas. Cancer 2004;105:35-43.

5 Jiang F, Yin Z, Caraway NP, et al. Genomic profiles in stage I primary non small cell lung cancer using comparative genomic hybridization analysis of cDNA microarrays. Neoplasia 2004;6:623-635.

$6 \mathrm{Li} \mathrm{R}$, Wang $\mathrm{H}$, Bekele $\mathrm{BN}$, et al. Identification of putative oncogenes in lung adenocarcinoma by a comprehensive functional genomic approach. Oncogene 2005 (advance online publication).

7 Jiang F, Caraway NP, Wang H, et al. Surfactant protein A gene deletion and prognostics for patients with stage I non-small cell lung cancer. Clin Cancer Res 2005; 11:5417-5424.

8 Griffin DK, Sanoudou D, Adamski E, et al. Chromosome specific comparative genome hybridisation for determining the origin of intrachromosomal duplications. J Med Genet 1998;35:37-41.

9 Jiang F, Lin F, Price R, et al. Rapid detection of IgH/ BCL2 rearrangement in follicular lymphoma by interphase fluorescence in situ hybridization with bacterial artificial chromosome probes. J Mol Diagn 2002;4: 144-149.

10 Forster T, Costa Y, Roy D, et al. Triple-target microarray experiments: a novel experimental strategy. BMC Genom 2004;5:13.

11 Oga A, Kong G, Ishii Y, et al. Preferential loss of 5q14-21 in intestinal-type gastric cancer with DNA aneuploidy. Cytometry 2001;46:57-62.

12 Dawson AJ, Putnam S, Schultz J, et al. Cryptic chromosome rearrangements detected by subtelomere assay in patients with mental retardation and dysmorphic features. Clin Genet 2002;62:488-494.

13 Fiegler H, Carr P, Douglas EJ, et al. DNA microarrays for comparative genomic hybridization based on DOPPCR amplification of BAC and PAC clones. Genes Chromosomes Cancer 2003;36:361-374. 